\title{
The Novel Roles of Neutrophils Via Opioid Peptides: Regulation of the Estrous Cycle and Pain
}

\author{
Yoshiro Kobayashi
}

Received: 21 August 2012/Accepted: 1 February 2013/Published online: 12 February 2013

(C) L. Hirszfeld Institute of Immunology and Experimental Therapy, Wroclaw, Poland 2013

\begin{abstract}
Neutrophils are excreted into the vaginal vault at metestrus during the estrous cycle, and this phenomenon has long been used to determine the phase of the estrous cycle. A much smaller number of neutrophils are also detected in the uterus and the ovary. Recently, we provided several lines of evidence supporting the notion that neutrophils infiltrate into the ovary to regulate the estrous cycle by opioid peptides. Upon inflammation, on the other hand, neutrophils infiltrate into the site of infection to suppress pain by opioid peptides. Thus, opioid peptides are key molecules by which neutrophils play a novel role in regulation of the pain and estrous cycle. In both cases, opioid peptides appear to be secreted by neutrophils stimulated with chemokines, such as MIP-2 and KC in mouse, corticotropin-releasing hormone and IL-1.
\end{abstract}

Keywords Neutrophils - Opioid peptides - Estrous cycle · Pain

\section{Introduction}

Neutrophils comprise approximately 60 and $10 \%$ of human and mouse peripheral blood leukocytes, respectively, and they circulate in the blood stream under physiological conditions. It is generally believed that neutrophils enter sites of infection or ischemia-reperfusion, where they execute a bacteria-killing function or cause tissue damage, respectively. However, even under

\section{Y. Kobayashi $(\bowtie)$}

Division of Molecular Medicine, Department of Biomolecular

Science, Faculty of Science, Toho University,

Chiba, Funabashi, Japan

e-mail: yoshiro@biomol.sci.toho-u.ac.jp physiological conditions, neutrophils are excreted into the vaginal vault at metestrus during the estrous cycle. This phenomenon has long been known since Stockard and Papanicolaou found in 1917 that in guinea pigs, the morphology of epithelial cells in the vagina changes in association with the estrous cycle, and subsequently in 1922, Long and Evans published a method for determining the stage of the estrous cycle by means of vaginal smears (Goldman et al. 2007). It should be noted here that in rats and mice, the estrous cycle is driven by a light/dark cycle. The cycle comprises four stages, namely proestrus, estrus, metestrus and diestrus, and the cycle is repeated every 4-5 days.

Excretion of neutrophils into the vaginal vault is reportedly mediated by MIP-2, one of the neutrophilselective chemokines in mice, which is expressed on epithelial cells in the vaginal vault (Sonoda et al. 1998). The role of neutrophils excreted into the vaginal vault, however, has been a matter of dispute, although it has been hypothesized that they may phagocytose and kill commensal bacteria. We thus initiated research to elucidate their role about seven years ago. Because it is well known that the administration of an anti-Gr-1 monoclonal antibody (anti-Gr-1 mAb) to mice causes depletion of neutrophils due to a high level of expression of Gr-1 antigen on neutrophils, we administered the mAb to determine its effect on vaginal smears, in which we found many cocci, as expected. To our surprise, however, the administration also caused blockading of the estrous cycle at diestrus as well as that of the cyclic change of steroid hormones, that is, estradiol and progesterone, although it has been widely accepted that the estrous cycle is driven by the hypothalamic-pituitary-ovarian axis (a central mechanism), and nobody has expected that neutrophils participate in regulation of the estrous cycle (Sasaki et al. 2009; a 
peripheral mechanism). We also noticed that the levels of steroid hormones gradually returned to normal even though mice were kept neutropenic (Sasaki et al. 2009). These findings suggest that neutrophils are involved in regulation of the estrous cycle and that there must be a backup mechanism independent of neutrophils. The backup mechanism is perhaps the same as a central mechanism described above (see "Summary and Future Direction"), although it is not known at present. Our subsequent study provided several lines of evidence supporting the notion that upon the entry of neutrophils into the ovaries, they secrete opioid peptides to regulate the estrous cycle (Sasaki et al. 2011).

Because neutrophils are reported to regulate pain through opioid peptides such as $\beta$-endorphin at inflammatory sites (Machelska and Stein 2006), I would like to summarize our results and compare these two phenomena in this review.

\section{The Mechanism Underlying Neutrophil-Dependent Regulation of the Estrous Cycle}

Although I stated in the introduction that the administration of an anti-Gr-1 mAb caused depletion of neutrophils, there has been a report that an anti-Gr-1 mAb also caused depletion of eosinophils in addition to neutrophils. Since female genital organs such as the vaginal vault and uterus contain not only neutrophils but also eosinophils, it is important to determine whether or not the administration of the anti-Gr-1 mAb also causes depletion of eosinophils. Under our conditions, however, the administration did not affect eosinophils in female genital organs, which was perhaps due to the difference in the conditions, namely normal mice in our study versus infected mice in other studies (Sasaki et al. 2009).

In order to elucidate the mechanism underlying neutrophil-dependent regulation of the estrous cycle, we presumed that the localization of neutrophils in female genital organs would provide a clue. Since we detected many neutrophils in the vaginal vault at metestrus but only a few in the uterus and the ovaries (Sasaki et al. 2009), we initially hypothesized that neutrophils entering the vagina and vaginal vault play a role in regulation in the estrous cycle (Sasaki et al. 2009). We then examined from which blood vessels neutrophils enter the female genital organs but we failed to obtain definitive results (Sasaki et al. unpublished). In the meantime, we came across publications reporting that opioid peptides regulate steroidogenesis in theca cells and granulose cells in the ovaries (Kaminski et al. 2003, 2004), and we finally hypothesized that neutrophils may secrete opioid peptides in the ovaries, thereby regulating the estrous cycle (Sasaki et al. 2011).
Because naloxone is well known as an opioid receptor antagonist, we predicted that the compound would block the estrous cycle. The results were exactly as we predicted (Sasaki et al. 2011). The estrous cycle length in ICR mice was $4.7 \pm 0.8$ days $(n=54)$, whereas those in anti-Gr-1 mAb-injected mice and naloxone-treated ones were prolonged to $7.7 \pm 2.3(n=67)$ and $7.9 \pm 2.0$ days $(n=7)$, respectively. The difference between control mice and antiGr-1 mAb-injected ones and that between control mice and naloxone-treated ones were statistically significant. Additionally, the estrogen and progesterone levels in plasma were affected in naloxone-treated mice in the same way as in anti-Gr-1 mAb-injected ones (Sasaki et al. 2011). It is noteworthy that when naloxone was administered only once at estrus, but not other phases, the estrous cycle was blocked (Sasaki et al. 2011), confirming the previous finding with anti-Gr-1 mAb that the estrus phase is the most critical among the four phases for neutrophil-dependent regulation of the estrous cycle (Sasaki et al. 2009). These results are consistent with the hypothesis that neutrophils may secrete opioid peptides in the ovaries at estrus, thereby regulating the estrous cycle.

Then, which type of opioid receptor(s) is involved in neutrophil-mediated regulation of estrous cycle? We thus employed type-specific receptor agonists to examine whether or not they shorten (normalize) the estrous cycle length in anti-Gr-1 mAb-injected mice. The administration of [D$\mathrm{Ala}^{2}, N$-MePhe ${ }^{4}$, Gly-ol ${ }^{5}$ ]-enkephalin (DAMGO), a $\mu$ opioid receptor agonist, to neutrophil-depleted mice shortened the cycle length to $6.0 \pm 0.7$ days $(n=5)$, the difference between anti-Gr-1 mAb-injected mice and DAMGO-administered, anti-Gr-1 mAb-injected ones being statistically significant (Sasaki et al. 2011). Such an effect was not observed with $(5 \alpha, 7 \alpha, 8 \beta)-(-)-N$-methyl- $N$-(7-(1pyrrolidinyl)-1-oxaspiro(4,5)dec-8-yl)benzenacetamide (U -69593), a $\kappa$ opioid receptor agonist, or [D-Pen ${ }^{2,5}$ ]enkephalin (DPDPE), a $\delta$ opioid receptor agonist (Sasaki et al. 2011). Endorphins and enkephalins bind to $\mu$ opioid receptors selectively, whereas dynorphins bind to $\kappa$ opioid receptors selectively. Enkephalins, on the other hand, bind to $\delta$ opioid receptors selectively. Therefore, these results suggest the possibility that $\beta$-endorphin derived from neutrophils is involved in regulation of the estrous cycle.

Opioid peptides are reportedly detected in leukocytes. For instance, proopiomelanocortin (POMC), a precursor of $\beta$-endorphin, was detected in approximately $40 \%$ rat peripheral blood neutrophils (Mousa et al. 2004). We detected POMC in approximately $35 \%$ mouse peripheral blood neutrophils and approximately $26 \%$ mouse blood neutrophils in the ovaries (Fig. 1A, Aa, Ab), but not in neutrophils that had infiltrated into the stroma of ovaries (Fig. 1C) (Sasaki et al. 2011). These results suggest that neutrophils are recruited to the ovaries by means of 
Fig. 1 Immunohistochemical detection of neutrophils and POMC in the ovaries. The ovaries were obtained at $6 \mathrm{pm}$ on the day of estrus, followed by preparation of paraffin sections. A section was stained with either anti-POMC antibodies (A, Aa, $\mathbf{A b}, \mathbf{C})$ or anti-Gr-1 mAb (B). B and $\mathbf{C}$ were serial sections. In panels $\mathbf{A a}$ and $\mathbf{A b}$, black and white arrows indicate positive and negative signals, respectively. Scale bars, $25 \mu \mathrm{m}$ (Sasaki et al. (2011); copyright $₫$ from the Journal of Immunology)
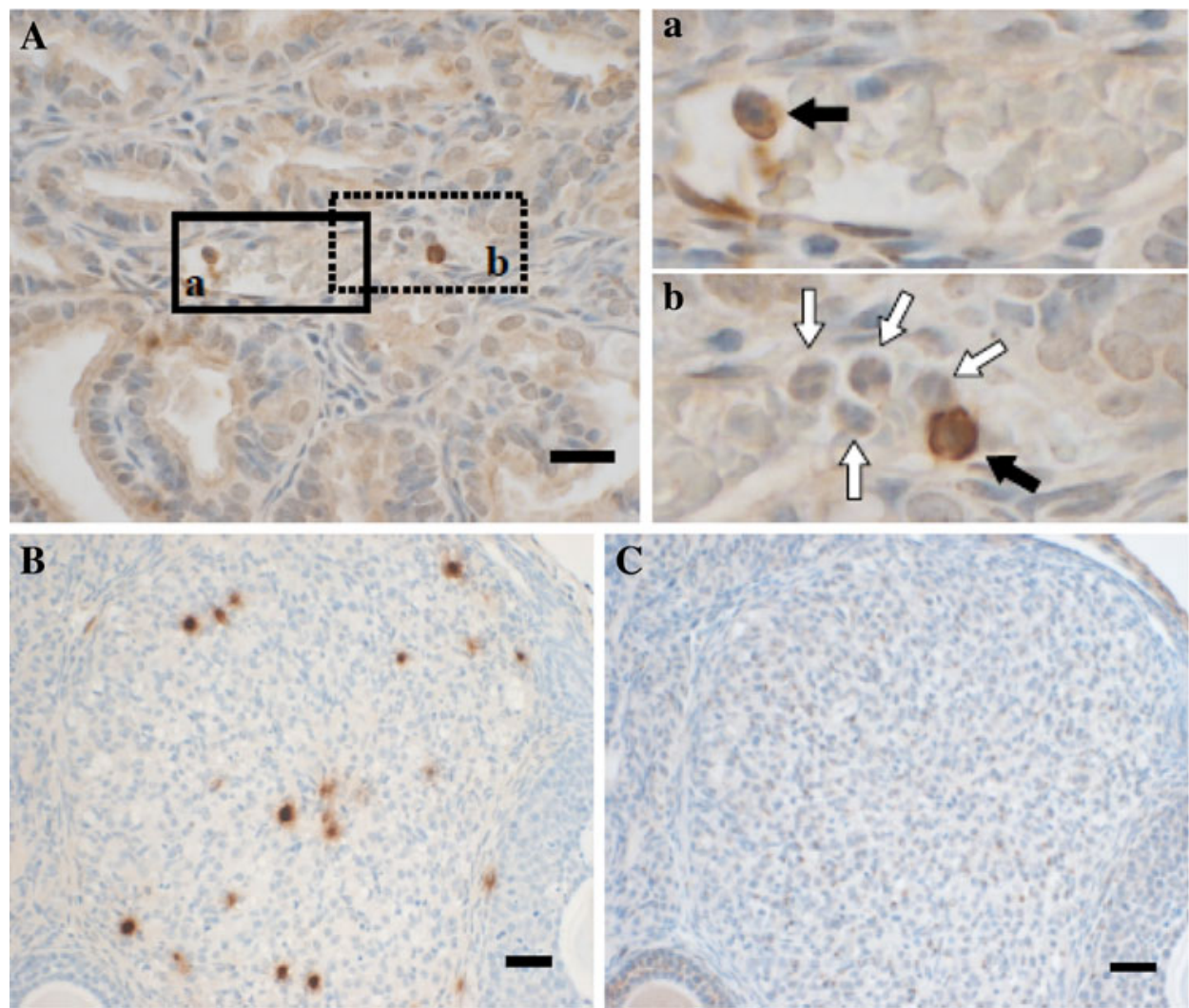

unknown chemotactic factor(s), and they are then activated to release opioid peptides. Since the administration of antibodies against MIP-2 or KC, another chemokine selective for neutrophils in mice, caused blockading of the estrous cycle (Sasaki et al. 2011), it is possible that MIP-2 and $\mathrm{KC}$ are involved in release of opioid peptides. The identification of the chemotactic factor(s) awaits further study.

Intravenous administration of MIP-2 also caused blockading of the estrous cycle as well as an increase in CD69 expression on peripheral blood neutrophils (Sasaki et al. 2011). Here, it should be noted that CD69 expression is a marker of the activation of leukocytes. We therefore hypothesized that the activation of neutrophils by MIP-2 caused secretion of opioid peptides in the peripheral blood, thereby abolishing their ability to regulate the estrous cycle. Indeed, the POMC signal was hardly detected in peripheral blood neutrophils of MIP-2-treated mice (Sasaki et al. 2011), corroborating the hypothesis. Since stress causes an increase in the plasma IL-8 level (Weik et al. 2008) as well as blockading of the menstrual cycle, there is a possibility that the effect of stress on the menstrual cycle can be explained by the above finding. This possibility is worthy of further study, because future research may lead to the development of a novel drug overcoming the effect of stress.
These findings allow us to propose our current model of neutrophil-dependent regulation of the estrous cycle (Fig. 2).

\section{Regulation of Pain by Neutrophil-Derived Opioid Peptides}

Upon injury or infection, inflammation ensues. A variety of molecules, including protons, ATP, neural peptides, prostaglandins, bradykinin, cytokines and chemokines, then cause pain, whereas leukocytes, including neutrophils, monocytes and lymphocytes, infiltrate into the site of inflammation and suppress the pain through the binding of opioid peptides to their receptors on peripheral sensory neurons. Neutrophils release opioid peptides at an early phase of inflammation, whereas monocytes and lymphocytes do so at a late phase of inflammation.

The regulation of pain by leukocyte-derived opioid peptides has been studied by the group of Drs. C. Stein and H. Machelska (2006). They obtained several lines of evidence supporting such a regulatory mechanism, as follows. (1) Opioid peptide receptors are expressed on peripheral sensory neurons. (2) The mRNAs and proteins of POMC, proenkephalin and prodynorphin are detected in leukocytes. (3) Leukocytes containing opioid peptides accumulate at 


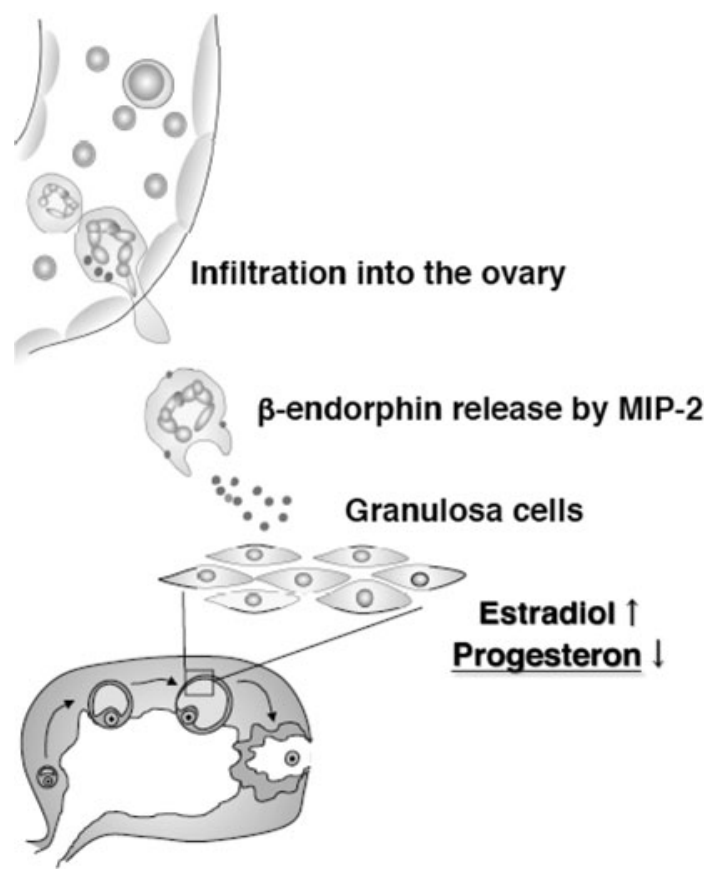

Fig. 2 Our model of neutrophil-dependent regulation of the estrous cycle

the site of inflammation. (4) Leukocytes release opioid peptides in vitro in response to appropriate stimuli such as corticotropin-releasing hormone (CRH), IL-1 and IL-8. (5) Administration of $\mathrm{CRH}$ to the site of inflammation decreases pain, whereas neutralization of $\mathrm{CRH}$ at the site of inflammation increases pain (Labuz et al. 2009; Machelska and Stein 2006; Rittner et al. 2006). (6) In CRH- or IL-1 $\beta$ induced pain inhibition, leukocytes apparently are the target, because depletion of granulocytes as well as blockade of chemokines (MIP-2 and KC) results in a significant reduction in opioid-containing cells and pain inhibition (Machelska and Stein 2006). (2) and (4) also support the neutrophil-dependent regulatory mechanism by which the estrous cycle is maintained.

Recently, Stein and his colleagues reported that formyl peptide receptors are involved in opioid peptide release in vivo in Freund's complete adjuvant-induced inflammation (Rittner et al. 2009). According to them, mycobacteria contain formyl peptides, which are released during bacterial lysis. This is perhaps the source of formyl peptides in this model of inflammation. There is a possibility, however, that MIP-2 and KC are also involved in the opioid peptide release in the model, because these chemokines are presumably produced in it.

Future studies should demonstrate that leukocytes at the site of inflammation indeed release opioid peptides and that these findings can be applied to clinical settings.
Possible Involvement of IL-1 and CRH in NeutrophilMediated Regulation of the Estrous Cycle

As described above, IL-1 and CRH are involved in neutrophil-mediated regulation of pain. These two molecules are generally associated with inflammation. In the mouse, however, the ovarian synthesis of IL- $1 \alpha$ and IL- $1 \beta$ mRNA was detected by in situ hybridization (Takacs et al. 1988). In the rat, immunoreactive IL- $1 \alpha$ was detected in the ovary at proestrus and estrus but not at diestrus (Nappi et al. 1994). Various ovarian cell types, such as the oocyte, granulosa and theca cells, have been identified as the sites of synthesis of IL-1 (Gerard et al. 2004). There is evidence for the involvement of ovarian IL-1 in the ovulation process and the regulation of ovarian steroidogenesis (Gerard et al. 2004). These considerations raise the possibility that IL-1 in these ovarian cell types may be involved in regulation of the estrous cycle.

Although neutrophils are known to contain IL-1 (Lloyd and Oppenheim 1992), there has been controversy about whether non-stimulated neutrophils produce IL-1. One study reported that only IL- 8 mRNA was detected in nonstimulated human neutrophils, whereas IL-1 $\beta$, IL- 6 and TNF- $\alpha$ mRNAs were detected after stimulation of neutrophils with lipopolysaccharide or zymosan (Altstaedt et al. 1996). However, the authors did not detect IL-1 $\beta$ protein in the culture supernatant even after stimulation (Altstaedt et al. 1996). Therefore, it is not known at present whether neutrophils infiltrated into the ovary produce IL-1. If the possibility is proven correct, then there is the possibility that IL-1 in neutrophils may also participate in regulation of the estrous cycle. Since IL-1 causes opioid peptide release (Machelska and Stein 2006), IL-1 may regulate the estrous cycle via opioid peptides.

CRH also reportedly regulates ovarian steroidogenesis (Murase et al. 2002), and CRH is present in immune cells, fibroblasts and vascular endothelium (Schaefer 2003). CRH is also detected in the ovary (Xu et al. 2007), and it affects steroidogenesis through IL-1 (Ghizzoni et al. 1997). Therefore, there is also the possibility that CRH may be involved in neutrophil-mediated regulation of estrous cycle via IL-1.

\section{Summary and Future Direction}

Neutrophils contain primary, secondary, tertiary and secretory granules, and the constituents of these granules are released during infiltration through the endothelial cell layer surrounding blood vessels into tissue (Soehnlein et al. 2009). Neutrophils first attach to the endothelial cells, and the secretory granules are then de-granulated into the 
lumen. Neutrophils then transmigrate through the endothelial cell layer, during which tertiary granules are degranulated. Neutrophils finally migrate into an infected site, during which primary and secondary granules are (partially) de-granulated. Opioid peptides ( $\beta$-endorphin and met-enkephalin) are contained in the same primary granules as myeloperoxidase (Rittner et al. 2008).

Regulation of pain by neutrophil-derived opioid peptides is regarded as an inflammatory response, whereas regulation of the estrous cycle by neutrophil-derived opioid peptides as a physiological process. Although IL-1, CRH and IL-8 appear to be involved in regulation of pain by neutrophils (Machelska and Stein 2006), IL-1 (Takacs et al. 1988), CRH (Xu et al. 2007) and MIP-2 (Sasaki et al. 2009) are also detected in the ovary even under physiological conditions. Consequently, it is possible that these molecules are responsible for opioid peptide release from neutrophils in both processes, and the difference between these two processes may be quantitative but not qualitative. Future studies should clarify the possibility.

Much more remains unresolved as to neutrophildependent regulation of the estrous cycle, when compared with neutrophil-dependent regulation of pain. For instance, (1) what is the physiological significance of the neutrophildependent regulatory mechanism for the estrous cycle? (2) what is the relationship between the neutrophil-dependent and neutrophil-independent mechanisms? In relation to the question (2), is the backup mechanism (neutrophil-independent mechanism) regulated by the hypothalamicpituitary-ovarian axis? Because we recently found a strain of mice that is deficient in the neutrophil-dependent regulatory mechanism for the estrous cycle (Sasaki et al. unpublished), future studies should provide clues as to these questions.

\section{References}

Altstaedt J, Kirchner H, Rink L (1996) Cytokine production of neutrophils is limited to interleukin-8. Immunology 89:563-568

Gerard N, Caillaud M, Martoriati A et al (2004) The interleukin-1 system and female reproduction. J Endocrinol 180:203-212

Ghizzoni L, Mastorakos G, Vottero A et al (1997) Corticotropinreleasing hormone $(\mathrm{CRH})$ inhibits steroid biosynthesis by cultured human granulosa-lutein cells in a CRH and interleukin-1 receptor-mediated fashion. Endocrinology 138:4806-4811
Goldman JM, Murr AS, Cooper RL (2007) The rodent estrous cycle: characterization of vaginal cytology and its utility in toxicological studies. Birth Defects Res B Dev Reprod Toxicol 80:84-97

Kaminski T, Siawrys G, Bogacka I et al (2003) The regulation of steroidogenesis by opioid peptides in porcine theca cells. Anim Reprod Sci 78:71-84

Kaminski T, Siawrys G, Bogacka I et al (2004) The influence of opioid peptides on steroidogenesis in porcine granulosa cells. Reprod Domest Anim 39:25-32

Labuz D, Schmidt Y, Schreiter A et al (2009) Immune cell-derived opioids protect against neuropathic pain in mice. J Clin Invest 119:278-286

Lloyd AR, Oppenheim JJ (1992) Poly's lament: the neglected role of the polymorphonuclear neutrophil in the afferent limb of the immune response. Immunol Today 13:169-172

Machelska H, Stein C (2006) Leukocyte-derived opioid peptides and inhibition of pain. J Neuroimmune Pharmacol 1:90-97

Mousa SA, Shakibaei M, Sitte N et al (2004) Subcellular pathways of beta-endorphin synthesis, processing, and release from immunocytes in inflammatory pain. Endocrinology 145:1331-1341

Murase M, Uemura T, Kondoh Y et al (2002) Role of corticotropinreleasing hormone in ovarian steroidogenesis. Endocrinology 18: 255-260

Nappi RE, Guo AL, Petraglia F et al (1994) Pituitary and ovarian interleukin-1 alpha content changes according to estrous cycle and acute stress exposure. Gynecol Endocrinol 8:259-264

Rittner HL, Labuz D, Schaefer M et al (2006) Pain control by CXCR2 ligands through $\mathrm{Ca} 2+$-regulated release of opioid peptides from polymorphonuclear cells. FASEB J 20:2627-2629

Rittner HL, Brack A, Stein C (2008) Pain and the immune system. Br J Anaesth 101:40-44

Rittner HL, Hackel D, Voigt P et al (2009) Mycobacteria attenuate nociceptive responses by formyl peptide receptor-triggered opioid peptide release from neutrophils. PLoS Pathog 5:e1000362

Sasaki S, Nagata K, Kobayashi Y (2009) Regulation of the estrous cycle by neutrophil infiltration into the vagina. Biochem Biophys Res Commun 382:35-40

Sasaki S, Tamaki Y, Nagata K et al (2011) Regulation of the estrous cycle by neutrophils via opioid peptides. J Immunol 187:774-780

Schaefer M (2003) Cytokines and peripheral analgesia. Adv Exp Med Biol 521:40-50

Soehnlein O, Weber C, Lindbom L (2009) Neutrophil granule proteins tune monocytic cell function. Trends Immunol 30:538-546

Sonoda Y, Mukaida N, Wang J et al (1998) Physiologic regulation of postovulatory neutrophil migration into vagina in mice by a C-X-C chemokine(s). J Immunol 160:6159-6165

Takacs L, Kovacs EJ, Smith MR et al (1988) Detection of IL-1 alpha and IL-1 beta gene expression by in situ hybridization. Tissue localization of IL-1 mRNA in the normal C57BL/6 mouse. J Immunol 141:3081-3095

Weik U, Herforth A, Kolb-Bachofen V et al (2008) Acute stress induces proinflammatory signaling at chronic inflammation sites. Psychosom Med 70:906-912

$\mathrm{Xu} \mathrm{J,} \mathrm{Xu} \mathrm{F,} \mathrm{Hannebold} \mathrm{JD} \mathrm{et} \mathrm{al} \mathrm{(2007)} \mathrm{Expression} \mathrm{and} \mathrm{role} \mathrm{of} \mathrm{the}$ corticotropin-releasing hormone/urocortin-receptor-binding protein system in the primate corpus luteum during the menstrual cycle. Endocrinology 148:5385-5395 